\title{
Analysis on Moving Capability Characteristics of Mechanism Based on Equivalent Transformation of Virtual Compound Joint
}

\author{
Jianguo Luo ${ }^{1}$, Zehao $\mathrm{Bu}^{2}$ and Jianyou $\mathrm{Han}^{3}$ \\ ${ }^{1}$ North China Institute of Science and Technology, Mechanical \& Electrical Department, Beijing, 101601 \\ ${ }^{2}$ North China Institute of Science and Technology, Graduate School, Beijing, 101601 \\ ${ }^{3}$ Beijing University of Science \& Technology, School of Mechanical Engineering, Beijing, 100083
}

\begin{abstract}
By introducing the theory of virtual compound joint and its characteristic parameters analysis method, the moving capability of the output terminal of the kinematic chain can be obtained by calculating and analyzing the instantaneous or global equivalent rotation angle domain and instantaneous or global equivalent translation domain of virtual compound joint. Analyzing the kinematic chain over 3 different cases base on its joint composition, then finding the solution of equivalent rotation angle domain or/and equivalent translation domain of compound joint. Six-degree-of-freedom series-connected robot is analyzed as an example, the corresponding quantified results under given conditions are obtained by using MATLAB, the moving capability characteristics of the series-connected robot such as global equivalent rotation angle domain and global equivalent translation domain are presented in a direct and clear way by planar six-dimensional topological graph, the degree of freedom, working space, singularity are obtained at the same time.
\end{abstract}

Keywords-virtual compound joint; equivalent transformation; equivalent rotation angle domain; equivalent translation domain; topological graph

\section{INTRODUCTION}

In 1955, Denavit and Hartenberg published a paper in "ASME Journal of Applied Mechanics", then people use the method in this paper represent and make model of robot, and it's motion equations are derived, which has become a standard representing and modeling method for robot [1].In recent years, there are a lot of studies based on D-H method and homogeneous coordinate transformation. Yang Jidong [2] studied a $\mathrm{H}$ parallel mechanism which with two degrees of freedom, getting its positive and inverse solutions of its position by utilizing kinematic superposition principle, evaluating its movement performance by utilizing the condition number of Jacobi matrix and analyzing its accessible working space. Han Yingying [3] established an extensible, ring-shaped constraint equations with multiple symmetric surfaces based on the D-H matrix transfer method. Shan Peng $[4,5]$ used D-H transformation matrix as the modeling tool, established a general posture equation of 6 degrees of freedom parallel machine, and the inverse kinematics equation is solved. Li Ruiqin [6] take a random branched chain of 3-RSR parallel robot as the research object, established a position relation matrix of moving platform against static platform by using D-H matrix. In the paper [7], it mentioned the utilizing of mathematical tools in the research of the mechanism has made great achievements, and will continue to play an important role in the future.

In this paper, we made some progress by introducing the virtual compound joint theory and characteristic parameter analysis method, the moving capability characteristics of output of kinematic chain can be obtained through the calculation of instantaneous or global equivalent rotation angle domain and translation domain of virtual compound joint. With the help of planar six-dimensional topological graph, a specific series robot with 6 degrees of freedom is analyzed, the results of mechanism space moving capability are obtained.

\section{BASIC DEFINITION OF COMPOUND JOINTS}

Virtual compound joint: Supposing that superposing some joints in the kinematic chain can create a new virtual joint, which has the moving ability of each joint, this is defined as virtual compound joint (compound joint for short).

Equivalent transformation of the compound joint: The equivalent translation of the kinematic chain which is replaced by compound joint along the three directions of basic coordinate system and equivalent rotation around the three axis of the based coordinate system are defined as the equivalent transformation of the compound joint, as shown in formula (1)

$$
F=\operatorname{trans}\left(x, p_{x}^{\prime}\right) \operatorname{trans}\left(y, p_{y}{ }^{\prime}\right) \operatorname{trans}\left(z, p_{z}^{\prime}\right) \operatorname{rot}\left(x, a^{m}\right) \operatorname{rot}\left(y, \beta^{m}\right) \operatorname{rot}\left(z, \gamma^{m}\right)
$$

The equivalent main rotation angle of the compound joint: The angles $\alpha^{m}, \beta^{m}, \gamma^{m}$ rotated around the three coordinate axis $x, y, z$ are defined as the equivalent main rotation angle.

Virtual link length: The distance from origin of basis coordinate system to the virtual link output end $P^{\prime}\left(p_{x}^{\prime}, p_{y}^{\prime}, p_{z}^{\prime}\right)$, the length $l_{e}=\sqrt{\left(p_{x}^{\prime}\right)^{2}+\left(p_{y}^{\prime}\right)^{2}+\left(p_{z}^{\prime}\right)^{2}}$ is defined as the length of the virtual link.

The equivalent rotation angle of the compound joint: The intersection of the compound joint equivalent main angle and vice angle constitute the equivalent angle $\alpha^{e}, \beta^{e}, \gamma^{e}$.

The equivalent translation of the compound joint: The intersection of the compound joint equivalent main translation 
and equivalence vice translation constitute the equivalent translation $p_{x}^{e}, p_{y}^{e}, p_{z}^{e}$.
The equivalent transformation matrix $\mathrm{T}=\mathrm{F}$, so the corresponding term in formula (2) is equal.

$$
\left[\begin{array}{cccc}
t_{11} & t_{12} & t_{13} & t_{14} \\
t_{21} & t_{22} & t_{23} & t_{24} \\
t_{31} & t_{32} & t_{33} & t_{34} \\
0 & 0 & 0 & 1
\end{array}\right]=\left[\begin{array}{cccc}
c \beta^{m} c \gamma^{m} & -c \beta^{m} s \gamma^{m} & s \beta^{m} & p_{x}{ }^{\prime} \\
s \alpha^{m} s \beta^{m} c \gamma^{m}+c \alpha^{m} s \gamma^{m} & -s \alpha^{m} s \beta^{m} s \gamma^{m}+c \alpha^{m} c \gamma^{m} & -s \alpha^{m} c \beta^{m} & p_{y} \\
-c \alpha^{m} s \beta^{m} c \gamma^{m}+s \alpha^{m} s \gamma^{m} & c \alpha^{m} s \beta^{m} s \gamma^{m}+s \alpha^{m} c \gamma^{m} & c \alpha^{m} c \beta^{m} & p_{z} \\
0 & 0 & 0 & 1
\end{array}\right]
$$

\section{CAlCUlation AND AnAlysis of COMPOUND JoInT}

A. Analysis of Equivalent Rotation Angle Domain of The Compound Joint

The first step: calculating and analyzing the equivalent main rotation angle by formula (2), the following are 5 cases for specific solution.

1) $\sin \beta^{m} \neq 0 \& \cos \beta^{m} \neq 0$, from formula (2) : $\sin \beta^{m}=t_{13}, \sin \alpha^{m}=-\frac{t_{23}}{\cos \beta^{m}}, \sin \gamma^{m}=-\frac{t_{12}}{\cos \beta^{m}}$

Result: $\alpha^{m}=\arcsin \left(-\frac{t_{23}}{\cos \beta^{m}}\right), \beta^{m}=\arcsin \left(t_{13}\right), \gamma^{m}=\arcsin \left(-\frac{t_{12}}{\cos \beta^{m}}\right)$

2) $\sin \beta^{m}=0 \& \cos \beta^{m}=1$, from formula (2) : $\cos \alpha^{m} \cos \beta^{m}=t_{33}, \cos \alpha^{m}=t_{33}, \sin \gamma^{m}=-\frac{t_{12}}{\cos \beta^{m}}=-t_{12}$

Result: $a^{m}=\arccos \left(t_{33}\right), \gamma^{m}=\arcsin \left(-t_{12}\right), \beta^{m}=0^{\circ} / 360^{\circ} /-360^{\circ}$

3) $\sin \beta^{m}=0 \& \cos \beta^{m}=-1$, from formula (2) : $\cos \alpha^{m} \cos \beta^{m}=t_{33}, \cos \alpha^{m}=-t_{33}, \sin \gamma^{m}=-\frac{t_{12}}{\cos \beta^{m}}=t_{12}$

Result: $a^{m}=\arccos \left(-t_{33}\right), \gamma^{m}=\arcsin \left(t_{12}\right), \beta^{m}=180^{\circ} /-180^{\circ}$

4) $\sin \beta^{m}=-1 \& \cos \beta^{m}=0$, from formula (2):

$$
\left\{\begin{array}{l}
-s \alpha^{m} c \gamma^{m}+c \alpha^{m} s \gamma^{m}=s\left(\gamma^{m}-\alpha^{m}\right)=t_{21} \\
c \alpha^{m} c \gamma^{m}+s \alpha^{m} s \gamma^{m}=c\left(\gamma^{m}-\alpha^{m}\right)=t_{31} \\
s \alpha^{m} s \gamma^{m}+c \alpha^{m} c \gamma^{m}=c\left(\alpha^{m}-\gamma^{m}\right)=t_{22} \\
-c \alpha^{m} s \gamma^{m}+s \alpha^{m} c \gamma^{m}=s\left(\alpha^{m}-\gamma^{m}\right)=t_{32}
\end{array}\right.
$$

From formula (3): $\gamma^{m}-\alpha^{m}=\operatorname{arctg}\left(t_{21} / t_{31}\right), 2$ cases as followed:

Case 1: Supposing $\gamma^{m}=0^{\circ}$, there is $\alpha^{m}=-\operatorname{arctg}\left(t_{21} / t_{31}\right)$, so that we can obtain the maximum and minimum value of $\alpha^{m}$, and $\beta^{m}=270^{\circ} /-90^{\circ}$.

Case 2: Supposing $\alpha^{m}=0^{\circ}$, there is $\gamma^{m}=\operatorname{arctg}\left(t_{21} / t_{31}\right)$, so that we can obtain the maximum and minimum value of $\gamma^{m}$, and $\beta^{m}=270 /-90^{\circ}$.

5) $\sin \beta^{m}=1 \& \cos \beta=0$, from formula (2):

$$
\left\{\begin{array}{c}
s \alpha^{m} c \gamma^{m}+c \alpha^{m} s \gamma^{m}=s\left(\alpha^{m}+\gamma^{m}\right)=t_{21} \\
-c \alpha^{m} c \gamma^{m}+s \alpha^{m} s \gamma^{m}=-c\left(\alpha^{m}+\gamma^{m}\right)=t_{31} \\
-s \alpha^{m} s \gamma^{m}+c \alpha^{m} c \gamma^{m}=c\left(\alpha^{m}+\gamma^{m}\right)=t_{22} \\
c \alpha^{m} s \gamma^{m}+s \alpha^{m} c \gamma^{m}=s\left(\alpha^{m}+\gamma^{m}\right)=t_{32}
\end{array}\right.
$$

From formula (4): $\gamma^{m}+\alpha^{m}=\operatorname{arctg}\left(-t_{21} / t_{31}\right), 2$ cases are as follow:

Case 1: Supposing $\gamma^{m}=0^{\circ}$ there is $\alpha^{m}=\operatorname{arctg}\left(-t_{21} / t_{31}\right)$, so that we can obtain the maximum and minimum value of $\alpha^{m}$, and $\beta^{m}=90^{\circ} /-270^{\circ}$.

Case 2: Supposing $\alpha^{m}=0^{\circ}$ there is $\gamma^{m}=\operatorname{arctg}\left(-t_{21} / t_{31}\right)$, so that we can obtain the maximum and minimum value of $\gamma^{m}$, and $\beta^{m}=90^{\circ} /-270^{\circ}$.

The instantaneous equivalent main rotation angle component domains obtained:

$$
\left\{\begin{array}{c}
\left\{\alpha^{m s}\right\}=\left[0, \alpha^{m}\right] /\left[\alpha^{m}, 0\right] \\
\left\{\beta^{m s}\right\}=\left[0, \beta^{m}\right] /\left[\beta^{m}, 0\right] \\
\left\{\gamma^{m s}\right\}=\left[0, \gamma^{m}\right] /\left[\gamma^{m}, 0\right]
\end{array}\right.
$$

The instantaneous main rotation angle component domains are a series of interval, union all the intervals to obtain the global main rotation angle component domain of the compound joint, as shown in formula (6).

$$
\left\{\begin{array}{l}
\left\{\alpha^{m c}\right\}=\left[\min \left(\alpha^{m}\right), \max \left(\alpha^{m}\right)\right] /\left[0, \max \left(\alpha^{m}\right)\right] /\left[\min \left(\alpha^{m}\right), 0\right] \\
\left\{\beta^{m c}\right\}=\left[\min \left(\beta^{m}\right), \max \left(\alpha^{m}\right)\right] /\left[0, \max \left(\beta^{m}\right)\right] /\left[\min \left(\beta^{m}\right), 0\right] \\
\left\{\gamma^{m c}\right\}=\left[\min \left(\beta^{m}\right), \max \left(\beta^{m}\right)\right] /\left[0, \max \left(\beta^{m}\right)\right] /\left[\min \left(\beta^{m}\right), 0\right]
\end{array}\right.
$$

The second step: calculating and analyzing the equivalent vice rotation angle by the equivalent transformation matrix $\mathrm{T}$.

1)The movement range of the mobile joint in the kinematic chain is $\left[0, a_{i}^{\max }\right], a_{i}(i=1,2, \cdots 6)$, when the extension length of mobile joint is 0 , the cosine angle between the virtual link axis and three axis of the base coordinate system $x, y, z$ is:

$$
\left\{\begin{array} { l } 
{ \alpha _ { 0 } ^ { v s } = \operatorname { a r c c o s } ( t _ { 1 4 } / \sqrt { t _ { 1 4 } { } ^ { 2 } + t _ { 2 4 } { } ^ { 2 } + t _ { 3 4 } { } ^ { 2 } } ) } \\
{ \beta _ { 0 } ^ { v s } = \operatorname { a r c c o s } ( t _ { 2 4 } / \sqrt { t _ { 1 4 } { } ^ { 2 } + t _ { 2 4 } { } ^ { 2 } + t _ { 3 4 } { } ^ { 2 } } ) } \\
{ \gamma _ { 0 } ^ { v s } = \operatorname { a r c c o s } ( t _ { 3 4 } / \sqrt { t _ { 1 4 } { } ^ { 2 } + t _ { 2 4 } { } ^ { 2 } + t _ { 3 4 } { } ^ { 2 } } ) }
\end{array} \quad ( 7 ) \quad \left\{\begin{array}{l}
\alpha_{1}^{v s}=\arccos \left(t_{14}{ }^{\prime} / \sqrt{t_{14}{ }^{\prime 2}+t_{24}{ }^{\prime 2}+t_{34}{ }^{\prime 2}}\right) \\
\beta_{1}^{v s}=\arccos \left(t_{24}{ }^{\prime} / \sqrt{t_{14}{ }^{\prime 2}+t_{24}{ }^{\prime 2}+t_{34}{ }^{\prime 2}}\right)(8) \\
\gamma_{1}^{v s}=\arccos \left(t_{34} / \sqrt{t_{14}{ }^{\prime 2}+t_{24}{ }^{\prime 2}+t_{34}{ }^{\prime 2}}\right)
\end{array}\right.\right.
$$


if the extension length is $a_{i}^{\max }(8)$

$$
\left\{\begin{array} { c } 
{ \{ \alpha ^ { v s } \} = [ \alpha _ { 0 } ^ { v s } , \alpha _ { 1 } ^ { v s } ] / [ \alpha _ { 1 } ^ { v s } , \alpha _ { 0 } ^ { v s } ] } \\
{ \{ \beta ^ { v s } \} = [ \beta _ { 0 } ^ { v s } , \beta _ { 1 } ^ { v s } ] / [ \beta _ { 1 } ^ { v s } , \beta _ { 0 } ^ { v s } ] ( 9 ) } \\
{ \{ \gamma ^ { v s } \} = [ \gamma _ { 0 } ^ { v s } , \gamma _ { 1 } ^ { v s } ] / [ \gamma _ { 1 } ^ { v s } , \gamma _ { 0 } ^ { v s } ] }
\end{array} \left\{\begin{array}{l}
\alpha^{v c}=\left[\min \left(\alpha^{v s} \mid L\right), \max \left(\alpha^{v s} \mid R\right)\right] \\
\beta^{v c}=\left[\min \left(\beta^{v s} \mid L\right), \max \left(\beta^{v s} \mid R\right)\right](10) \\
\gamma^{v c}=\left[\min \left(\gamma^{v s} \mid L\right), \max \left(\gamma^{v s} \mid R\right)\right]
\end{array}\right.\right.
$$

The instantaneous vice rotation angle component domain, as shown in formula (9).

2) The instantaneous vice rotation angle component domain is a series of instantaneous vice rotation angle component domain $\left\{\alpha^{v s}\right\},\left\{\beta^{v s}\right\},\left\{\gamma^{v s}\right\}$, merging all the interval, we can get new interval is the global main translation component domain, as shown in formula (10).

3) Merging the instantaneous/ global main rotation angle component domain and instantaneous/ global vice rotation angle component domain, the corresponding instantaneous / global equivalent rotation angle domain are obtained.

$$
\left\{\begin{array} { l } 
{ \alpha ^ { e s } = \alpha ^ { m s } \cup \alpha ^ { v s } } \\
{ \beta ^ { e s } = \beta ^ { m s } \cup \beta ^ { v s } } \\
{ \gamma ^ { e s } = \gamma ^ { m s } \cup \gamma ^ { v s } }
\end{array} \quad ( 1 1 ) \quad \left\{\begin{array}{l}
\alpha^{e c}=\alpha^{m c} \cup \alpha^{v c} \\
\beta^{e c}=\beta^{m c} \cup \beta^{v c} \\
\gamma^{e c}=\gamma^{m c} \cup \gamma^{v c}
\end{array}\right.\right.
$$

\section{B. Calculation and Analysis of Equivalent Translation} Domain of The Compound Joint

The third step: Utilizing the results from first step and the equivalent transformation matrix $\mathrm{T}$ to calculate the equivalent translation domain. Two cases as followed:

Case 1: when $\mathrm{T}[1,4] \mathrm{T}[2,4], \mathrm{T}[3,4]$ contain variable parameter $a_{i}(i=1,2, \cdots 6)$.

1) When $a_{i}$ takes the maximum or minimum value, the instantaneous main translation component domain $\left\{P_{x}^{m s}\right\},\left\{P_{y}^{m s}\right\},\left\{P_{z}^{m s}\right\}$, as shown in formula (13).

$$
\left\{\begin{array} { l } 
{ P _ { x } ^ { m s } = [ \operatorname { m i n } ( t _ { 1 4 } ) , \operatorname { m a x } ( t _ { 1 4 } ) ] } \\
{ P _ { y } ^ { m s } = [ \operatorname { m i n } ( t _ { 2 4 } ) , \operatorname { m a x } ( t _ { 2 4 } ) ] ( 1 3 ) } \\
{ P _ { z } ^ { m s } = [ \operatorname { m i n } ( t _ { 3 4 } ) , \operatorname { m a x } ( t _ { 3 4 } ) ] }
\end{array} \left\{\begin{array}{l}
P_{x}^{m c}=\left[\min \left(P_{x}^{m s} \mid L\right), \max \left(P_{x}^{m s} \mid R\right)\right] \\
P_{y}^{m c}=\left[\min \left(P_{y}^{m s} \mid L\right), \max \left(P_{y}^{m s} \mid R\right)\right](14) \\
P_{z}^{m c}=\left[\min \left(P_{z}^{m s} \mid L\right), \max \left(P_{z}^{m s} \mid R\right)\right]
\end{array}\right.\right.
$$

2) When the mobile joint takes the maximum or minimum value in its corresponding range of movement, the equivalent link length is a variable value in a series of instantaneous main translation component domain $\left\{P_{x}^{m s}\right\},\left\{P_{y}^{m s}\right\},\left\{P_{z}^{m s}\right\}$, merging all intervals can obtain the global main translation component domain $\left\{P_{x}^{m c}\right\},\left\{P_{y}^{m c}\right\},\left\{P_{z}^{m c}\right\}$, as shown in formula (14).

3) The equivalent link of compound joint rotating $\alpha, \beta, \gamma$ around the based $x, y, z$, the terminal point of equivalent link move from $p_{x}^{\prime}, p_{y}^{\prime}, p_{z}^{\prime}$ to $p_{x}^{\prime \prime}, p_{y}^{\prime \prime}, p_{z}^{\prime \prime}$, the interval formed by old and new points is the instantaneous vice translation component domain $\left\{P_{x}^{v s}\right\},\left\{P_{y}^{v s}\right\},\left\{P_{z}^{v s}\right\}$, as shown in formula (15).

$$
\left\{\begin{array} { l } 
{ P _ { x } ^ { v s } = [ p _ { x } { } ^ { \prime } , p _ { x ^ { \prime } } { } ^ { \prime \prime } ] / [ p _ { x } { } ^ { \prime \prime } , p _ { x } { } ^ { \prime } ] } \\
{ P _ { y } ^ { v s } = [ p _ { y } , p _ { y } { } ^ { \prime } ] / [ p _ { y } { } ^ { \prime } , p _ { y } ] ( 1 5 ) } \\
{ P _ { z } ^ { v s } = [ p _ { z } , p _ { z } { } ^ { \prime \prime } ] / [ p _ { z } { } ^ { \prime \prime } , p _ { z } ] }
\end{array} \left\{\begin{array}{l}
P_{x}^{v c}=\left[\min \left(p_{x}{ }^{\prime}\right) / \min \left(p_{x}{ }^{\prime \prime}\right), \max \left(p_{x}{ }^{\prime}\right) / \max \left(p_{x}{ }^{\prime \prime}\right)\right] \\
P_{y}^{v c}=\left[\min \left(p_{y}{ }^{\prime}\right) / \min \left(p_{y}{ }^{\prime \prime}\right), \max \left(p_{y}{ }^{\prime}\right) / \max \left(p_{y}{ }^{\prime \prime}\right)\right](16) \\
P_{z}^{v c}=\left[\min \left(p_{z}{ }^{\prime}\right) / \min \left(p_{z}{ }^{\prime \prime}\right), \max \left(p_{z}{ }^{\prime}\right) / \max \left(p_{z}{ }^{\prime \prime}\right)\right]
\end{array}\right.\right.
$$

4) The equivalent link of compound joint rotated a series of equivalent rotation angle $\alpha, \beta, \gamma$ around $x, y, z$, the terminal point move from $p_{x}{ }^{\prime}, p_{y}{ }^{\prime}, p_{z}{ }^{\prime}$ to a series of new position $p_{x}{ }^{\prime}, p_{y}{ }^{\prime \prime}, p_{z}{ }^{\prime \prime}$, a series of interval formed by the old and new points, merging all intervals can get an interval with maximum and minimum, this new interval is the global vice translation component domain $\left\{P_{x}^{v c}\right\},\left\{P_{y}^{v c}\right\},\left\{P_{z}^{v c}\right\}$, as shown in formula (16).

5) Merging the instantaneous/ global main translation component domain and instantaneous/ global vice translation component domain, get the corresponding instantaneous / global equivalent translation domain.

$$
\left\{\begin{array} { l } 
{ P _ { x } ^ { e s } = P _ { x } ^ { m s } \cup P _ { x } ^ { v s } } \\
{ P _ { y } ^ { e s } = P _ { y } ^ { m s } \cup P _ { y } ^ { v s } } \\
{ P _ { z } ^ { e s } = P _ { z } ^ { m s } \cup P _ { z } ^ { v s } }
\end{array} \quad \text { (17) } \left\{\begin{array}{l}
P_{x}^{e c}=P_{x}^{m c} \cup P_{x}^{v c} \\
P_{y}^{e c}=P_{y}^{m c} \cup P_{y}^{v c} \\
P_{z}^{e c}=P_{z}^{m c} \cup P_{z}^{v c}
\end{array}\right.\right.
$$

Case 2: if $\mathrm{T}[1,4], \mathrm{T}[2,4], \mathrm{T}[3,4]$ do not contain variable $a_{i}(i=1,2, \cdots 6)$, the equivalent main translation domain does not exist, but the equivalent vice translation domain exists. The solution method only includes the (3), (4), (5) parts in first case.

\section{Topological Analysis of Characteristic Parameters}

The fourth step: bases on the first three steps, we can obtain the global equivalent rotation angle domain (divided into global main rotation angle component domain and global vice rotation angle component domain) and the global equivalent translation domain (divided into the global main translation component domain and the global vice translation component domain) under specific parameters condition, and present it by planar six dimensional topological graph. The specific method is in literature [8].

\section{CASE ANALysis}

As shown in Figure 1, the robot has six degrees of freedom, all joints are rotation joints. According to the D-H representation, build coordinate systems. Suppose that the range $\theta_{i}(i=1,2, \cdots 6)$ is $\left[25^{\circ}, 30^{\circ}\right], a_{2}=a_{3}=200 \mathrm{~mm}, a_{4}=100 \mathrm{~mm}$. The calculation process:

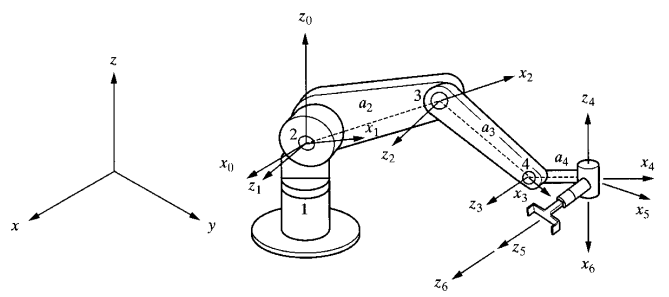

FIGURE I. SERIES-CONNECTED ROBOT WITH 6 DEGREES OF FREEDOM AND ITS REFERENCE COORDINATE SYSTEM 
1) Establish robot coordinate systems, supposing the joint 2, 3 and 4 are in the same plane, their $d_{n}$ values are 0.

2) Give the homogeneous transformation matrix between two adjacent joints.

3) Due to $\mathrm{T}[1,4], \mathrm{T}[2,4], \mathrm{T}[3,4]$, whose $a_{i}$ are a fixed value parameter, the instantaneous vice rotation angle component domain, global vice rotation angle component domain and the equivalent main translation do not exist.

4) Calculating $T=A_{1} A_{2} A_{3} A_{4} A_{5} A_{6}$, the instantaneous equivalent rotation angle domain and the global equivalent rotation angle domain can be obtained by using the formula (2) and the method in section 3 of this paper.

5) Instantaneous vice translation component domain can be obtained through previous third step, based on that the global vice translation component domain can be obtained.

The above calculation can be processed by MATLAB programming. The results are showed in figure 2 and 3.The mechanism has 3 rotation degrees of freedom according to the result of 3 rotation characteristic elements in topological representation lines. It has 3 translation degrees of freedom according to the result of 3 translation characteristic elements. These 3 translation degrees of freedom are accompanied translation (vice translation) formed by the kinematic chain rotation pair interaction, so the output end has 6 degrees of freedom.

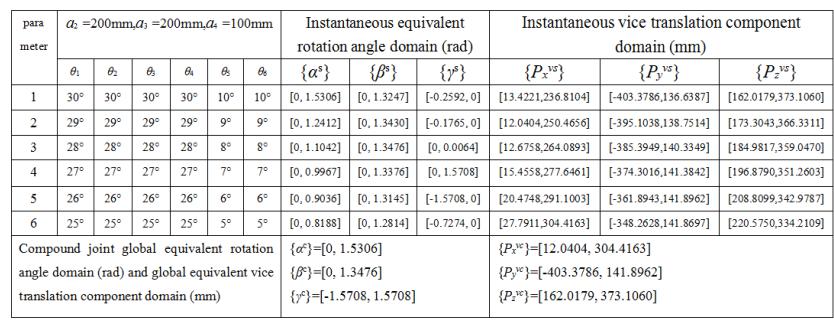

FIGURE II. THE PARAMETERS OF JOINTS IN KINEMATIC CHAIN AND THE OUTPUT RESULT OF COMPOUND JOINT

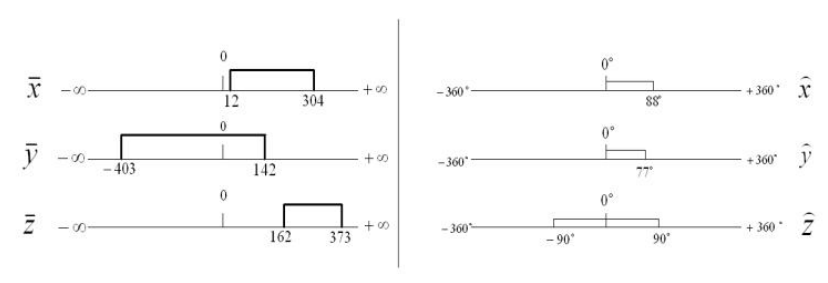

FIGURE III. PLANAR SIX-DIMENSIONAL TOPOLOGICAL REPRESENTATION

\section{CONCLUSION}

We draw some conclusions by introducing the virtual compound joint theory and characteristic parameter analysis method and the conclusions are as follows:

1) The homogeneous total transformation of kinematic chain can be replaced by equivalently transformation of virtual compound joint. The element in homogeneous transformation matrix can find a corresponding element in virtual compound joint. The moving capability characteristics of kinematic chain output end can be obtained by calculating the compound joint instantaneous / global equivalent rotation angle domain, instantaneous / global equivalent translation domain.

2) The moving capability characteristics parameters of the virtual compound joint kinematic chain can be presented by using planar six-dimensional topological graph, the degree of freedom, working space and singularity of kinematic chain output end can be obtained too.

3) The research gives the theory and method of general application on the analysis of mechanism degree of freedom, working space and singularity. Not only enrich and improve the existing mechanism research theories, but also play a key role in promoting the invention of new mechanism and accelerating the existing mechanism to be putted into practical engineering.

\section{ACKNOWLEDGMENT}

This research was financially supported by the HeBei Province Science and Technology Foundation (QN2017410) and NCIST Foundation (No. 3142015023).

\section{REFERENCE}

[1] HUANG Zhen, ZHAO Yongsheng, ZHAO Tieshi. Higher spatial mechanics[M]. Beijing: Higher Education Press, 2006

[2] YANG Jidong, YANG Zhongshan, LIU Dong, et al. Kinematics analysis of $\mathrm{H}$ parallel mechanism and experimental precision interpolation control experiment [J]. Journal of agricultural machinery, 2014, 45 (11): 325-329.

[3] HAN Yingying, YUAN Ru, WANG Sanmin. The D-H matrix transfer method for the configuration design of ring deployable mechanism [J]. Journal of Northwestern Polytechnical University, 2012,30 (5): 796-801.

[4] SHAN Peng, XIE Liyang, TIAN Wanlu, et al. Posture equation and dynamics reverse solution of Stewart type parallel machine tools based on D-H transformation matrix[J]. Mechanical Design, 2009, 26 (5): $15-18$.

[5] SHAN Peng, XIE Liyang, TIAN Wanlu, et al. Modeling of position and pose error of Stewart machine tool based on D-H transformation matrix[J]. Journal of Mechanical Engineering, 2010, 46 (17): 186-191.

[6] LI Ruiqin, YANG Bin. Error modeling of 3-RSR parallel robot based on D-H matrix $[\mathrm{J}]$. Journal of Measurement Science and Instrumentation, 2014,5(3): 53-59.

[7] WANG Guobiao, LIU Xinjun. Preliminary discussion on modern mathematics learning effect and influence of [J]. Chinese Journal of mechanical engineering in institutions, 2013,49 (3): 1-9.

[8] Jianguo Luo, Jianyou Han. Universal method on analysis of degree and working space and singularity of mechanism[J]. International Journal of Simulation Systems, Science\&Technology, 2016, Vol17(30):4.1-4.6. 